\title{
EZH2 negatively regulates PD-L1 expression in hepatocellular carcinoma
}

\author{
Gang Xiao 1,2,3†, Li-Lian Jin ${ }^{4 \dagger}$, Chao-Qun Liu ${ }^{1,2,3}$, Yong-Chun Wang ${ }^{1}$, Ya-Ming Meng ${ }^{3,4}$, Zhong-Guo Zhou ${ }^{5}$, \\ Jing Chen ${ }^{1}$, Xing-Juan $\mathrm{Yu}^{1}$, Yao-Jun Zhang ${ }^{5}$, Jing $\mathrm{Xu}^{1 *}$ and Limin Zheng ${ }^{1,3^{*}}$ (D)
}

\begin{abstract}
Background: Accumulating studies suggest that targeting epigenetic modifications could improve the efficacy of tumor immunotherapy; however, the mechanisms underlying this phenomenon remain largely unknown. Here, we investigated the ability of the epigenetic modifier, enhancer of zeste 2 polycomb repressive complex 2 subunit $(\mathrm{EZH} 2)$, to regulate the expression of immune checkpoint inhibitor, programmed death-1 ligand 1 (PD-L1) in hepatocellular carcinoma (HCC).

Methods: Immunohistochemistry and multiplex immunofluorescence staining were performed to analyze the expression and correlation of EZH2 and PD-L1 in HCC tissues. Immunoblotting, quantitative real-time PCR, flow cytometry, chromatin immunoprecipitation, and dual-luciferase reporter gene assays were performed to evaluate the regulatory roles of EZH2 on PD-L1 expression.

Results: In vitro cell experiments revealed that EZH2 negatively regulated the PD-L1 expression of hepatoma cell lines in IFNy-dependent manner. Mechanistic studies demonstrated that EZH2 could suppress PD-L1 expression by upregulating the H3K27me3 levels on the promoters of CD274 (encoding PD-L1) and interferon regulatory factor 1 (IRF1), an essential transcription factor for PD-L1 expression, without affecting the activation of the IFNY-signal transducer and activator of transcription 1 (STAT1) pathway. Clinical samples from HCC patients with immuneactivated microenvironments showed negative correlations between EZH2 and PD-L1 expression in hepatoma cells. Multivariate Cox analysis demonstrated that the combination of EZH2 and PD-L1 was an independent prognostic factor for both OS and RFS for patients with HCC.

Conclusions: The epigenetic modificator EZH2 can suppress the expression of immune checkpoint inhibitor PD-L1 by directly upregulating the promoter H3K27me3 levels of CD274 and IRF1 in hepatoma cells, and might serve as a potential therapeutic target for combination of immunotherapy for immune-activated HCC.
\end{abstract}

Keywords: EZH2, PD-L1, Epigenetics, Immunotherapy

\section{Background}

Cancer immunotherapy has become an important approach to harness the immune system to fight against cancer cells [1-3]. The use of immune checkpoint blockade to induce and restore -immune activation has produced durable clinical responses in the treatment of various tumor types; however, the response rates are still low [4-8]. Increasing evidence has demonstrated the

\footnotetext{
* Correspondence: xujing@sysucc.org.cn; zhenglm@mail.sysu.edu.cn

${ }^{\dagger}$ Gang Xiao and Li-Lian Jin contributed equally to this work.

${ }^{1}$ Collaborative Innovation Center of Cancer Medicine, State Key Laboratory of Oncology in South China, Sun Yat-sen University Cancer Center, Sun Yat-sen University, Guangzhou 510060, People's Republic of China

Full list of author information is available at the end of the article
}

immune-regulatory properties of epigenetic modulators in some preclinical models and in patients with advanced tumors, thus suggesting a rationale for combining epigenetics and immunotherapy [9-11]. However, the underlying mechanisms by which epigenetic modifiers influence the efficacy of immunotherapy are poorly understood.

As a key component of polycomb repressor complex 2 (PRC2), enhancer of zeste 2 polycomb repressive complex 2 subunit (EZH2) mediates trimethylation on histone 3 lysine 27 (H3K27me3) and gene silencing, and is involved in various biological processes $[12,13]$. Pathological activation of EZH2 histone methyltransferase (HMT) has

(c) The Author(s). 2019 Open Access This article is distributed under the terms of the Creative Commons Attribution 4.0 International License (http://creativecommons.org/licenses/by/4.0/), which permits unrestricted use, distribution, and 
been observed in tumor transcription programs [14], which influence cell growth [15], survival [16, 17], and metastasis $[18,19]$. As a potential target for cancer therapy, the therapeutic effects of EZH2 inhibitors are generally interpreted as the consequence of direct reduction of tumor cells (TCs) [20]. Recently, studies have revealed that EZH2 can also contribute to both local and systemic antitumor immunity [21]. For example, EZH2 can affect $\mathrm{CD}^{+} \mathrm{T}$ cell-mediated anti-tumor immunity by regulating the expression of Th-1 chemokines [22, 23] or the infiltration of myeloid-derived suppressor cells (MDSCs) [24, 25]. These data suggested that EHZ2, as an important modifier, could link epigenetic regulation and immune function; however, the mechanisms underlying this phenomenon in human tumors require further explanation.

Lack of programmed death-1 ligand 1 (PD-L1) expression is an important mechanism of resistance to the anti-PD-1/PD-L1 therapies [26]. Thus, many studies have attempted to determine the biological and clinical factors involved in PD-L1 expression [27-31]. Our recent study [32] revealed that expression pattern of PD$\mathrm{L} 1$ in TCs or monocytes/macrophages $(\mathrm{Mo} / \mathrm{M} \phi \mathrm{s})$ is a predictive biomarker in patients with hepatocellular carcinoma (HCC). The active immune milieu is associated with PD-L1 expression on M $\phi \mathrm{s}$; whereas, tumorexpressing PD-L1 may be hampered by certain cellintrinsic modifications [32]. Given the role of EZH2 in tumor immunity, we speculated that it would have a regulatory effect on PD-L1 expression in HCC.

In the present study, we provided evidence from clinical samples and in vitro cellular experiment that hepatoma-intrinsic EZH2 represses the expression of PD-L1. The results showed that EZH2 regulates the expression of PD-L1 through the epigenetic machinery, and thus could serve as a potential therapeutic target in combination with anti-PD-L1 immunotherapy.

\section{Methods}

\section{Patients and specimens}

Formalin-fixed, paraffin-embedded tissue from 386 patients with pathologically confirmed hepatocellular carcinoma (HCC), who had all received resection of the tumors at the Sun Yat-sen University Cancer Center between 2006 and 2010, were enrolled as previously described [32]. All samples were anonymously coded in accordance with local ethical guidelines (as requested by the Declaration of Helsinki), with written informed consent and using a protocol approved by the Review Board of Sun Yat-sen University Cancer Center. Overall survival (OS) was defined as the interval between surgery and death or between surgery and the last observation for the surviving patients. Relapse-free survival (RFS) was defined as the interval between surgery and the first of recurrence or death, or between surgery and the last observation for patients without recurrence. Tissues were used to construct a tissue microarray (TMA) as described previously [32]. A total of 386 patients who had complete OS and RFS information were used for the survival analysis. The clinical characteristics of all the patients are summarized in Additional file 2: Table S1.

\section{Immunostaining and image analysis}

Immunostaining and image analysis were conducted according to our previous reports [32]. In brief, TMA sections were dewaxed in xylene, rehydrated through a decreasing ethanol series, and then placed in $0.3 \% \mathrm{H}_{2} \mathrm{O}_{2}$ to diminish the activity of endogenous peroxidase. The sections were then heated for antigen retrieval. Following incubation with rabbit anti-human EZH2 (BD Transduction Laboratories, BD Biosciences, San Jose, CA, USA), immunostaining was performed using the EnVision Detection System (DakoCytomation, Carpinteria, CA, USA) following the manufacturer's instructions. Sections were counter-stained with hematoxylin. Image acquisition was performed using an Eclipse advanced research microscope (Nikon, Melville, NY, USA).

For multiplex immunofluorescence staining of EZH2, PD-L1 (clone: E1L3N ${ }^{\mathrm{m}}$; Cell Signaling Technology, Danvers, MA, USA) and CD68 (DakoCytomation), Tyramide Signal Amplification (TSA) Plus Fluorescence Kits (PerkinElmer, Foster City, CA, USA) combined with immunohistochemistry (IHC) was used. To obtain multispectral images, the stained slides were scanned using the Vectra System (PerkinElmer). The definition of PD-L1 positive expression was the same as that described previously [32]. For colocalization analysis, images were acquired using a laser confocal microscope (Olympus, Essex, UK) and analyzed using FV10-ASW Viewer software (Olympus).

The expression of EZH2 was determined by nuclear EZH2 expression on tumor cells and immunohistochemical scoring of EZH2 was analyzed using the Inform software (PerkinElmer) with the modified Histo-score (H-score), which involves assessing both the intensity of staining (graded as non-staining-0, weak-1, median-2 or strong-3) and the percentage of positive cells (Additional file 1: Figure S1). The range of possible scores was from 0 to 300 , quantified by $\mathrm{H}$-score. The correlation of EZH2 and PD-L1 expression was analyzed by $X^{2}$ test. The cutoff value for the $\mathrm{H}$-score was set at 35 with the minimum $P$ value to categorize the samples into EZH2 high or low groups.

\section{Cells}

The human hepatoma cell lines PLC/PRF/5, Huh7, and Hep3B used in this study were purchased from the American Type Culture Collection (Manassas, VA, 
USA). PLC/PRF/5 cells were cultured in Roswell Park Memorial Institute (RPMI) 1640 medium, and Huh7 and Hep3B cell lines were cultured in Dulbecco's modified Eagle medium (DMEM) supplemented with 10\% fetal bovine serum at $37^{\circ} \mathrm{C}$ and $5 \% \mathrm{CO}_{2}$. Hepatoma cells were treated with recombinant interferon gamma (IFNY) (Sino Biological Inc.), DZNep (MedChemExpress, Monmouth Junction, NJ, USA), or GSK-126 (MedChemExpress) for different times and at different concentrations.

Monocytes were selected from peripheral blood mononuclear cells using anti-CD14 magnetic beads (Miltenyi Biotec, Bergisch Gladbach, Germany) as described previously [33].

\section{RNA interference assay}

Hepatoma cells were transfected with small interfering RNAs (siRNAs) using Lipofectamine ${ }^{\circ}$ RNAiMAX Reagent (Invitrogen, Waltham, MA, USA). Reverse transfection was performed according to the manufacturer's instruction manual. The sequences of the siRNAs are listed in Additional file 2: Table S2.

\section{Flow cytometry}

Cells were collected by $0.25 \%$ trypsin digestion, and incubated with Phycoerythrin (PE) conjugated PD-L1 or isotype antibodies (eBioscience, San Diego, CA, USA). The cells were then subjected to flow cytometry.

\section{Quantitative real-time PCR (qPCR)}

Total RNA was isolated from cultured cells using TRIZOL (Invitrogen). Reverse transcription and real-time $\mathrm{PCR}$ were then performed using $5 \times$ All-In-One RT MasterMix (Applied Biological Materials, Richmond, Canada) and a SYBR green real-time PCR kit (Toyobo, Osaka, Japan). Relative quantification was calculated according to the comparative $\mathrm{Ct}$ method with normalization to the expression of GAPDH (encoding glyceraldehyde-3phosphate dehydrogenase). The primers used are listed in Additional file 2: Table S3.

\section{Immunoblotting analysis}

Cells were washed in phosphate-buffered saline (PBS) and suspended in Radioimmunoprecipitation assay (RIPA) buffer (Pierce, Rockford, IL, USA). Supernatant protein concentrations were determined using a BCA protein assay kit (Pierce). Supernatant samples were resolved by $10 \%$ or $15 \%$ SDS-PAGE depending on the sizes of target proteins, transferred to Immobilon-P polyvinylidene fluoride (PVDF) membranes (Millipore, Billerica, MA, USA) using electroblotting, and then probed with primary antibodies. Membranes were then incubated with horseradish peroxidase-conjugated secondary antibodies. The signals from the immunoreactive proteins were detected using the ECL reagent
(Millipore). The information about the antibodies is listed in Additional file 2: Table S4.

\section{Dual-luciferase reporter assay}

Huh7 and Hep3B cells pre-transfected with siRNAs, IFN $\gamma$, or not, were cotransfected with the pGL3-PD-L1 promoter-luc reporter or pGL3-basic control vectors. pRL-TK was used as an internal control. Cell lysates were harvested for the dual-luciferase assay, which was performed according to the manufacturer's instructions (Promega, Madison, WI, USA). The primers used are listed in Additional file 2: Table S5.

\section{Construction of Hep3B-shEZH2 cells}

To generate a cell line with the stable knockdown of $E Z H 2$, lentiviral plasmids carrying a short hairpin RNA (shRNA) targeting EZH2 (VectorBuilder Inc., Shenandoah, TX, USA) were transfected to $293 \mathrm{~T}$ cells together with plasmids PMD2.G and pSPAX2 using Lipofectamine 3000 (Invitrogen). After $48 \mathrm{~h}$, culture supernatants were collected, passed through $0.45-\mu \mathrm{m}$ filters, and mixed with fresh media (1:1) and polybrene $(8 \mu \mathrm{g} / \mathrm{ml})$ to infect Hep3B cells. Cells infected with shEZH2 or control vectors were designated as Hep3B-shEZH2 and Hep3B-vector stable cell lines respectively, and were established using $1 \mu \mathrm{g} / \mathrm{ml}$ puromycin selection. The shRNA-targeted regions in EZH2 were at nt 784-804.

\section{Plasmids}

To construction of PD-L1 overexpression plasmid, $E Z H 2$-silenced Hep3B cells were first treated with IFNY for $24 \mathrm{~h}$. Then, mRNA was extracted from these cells, and reverse transcription PCR was performed to obtain cDNA, which was used as template to amplify the coding sequence (CDS) of CD274 (encoding PD-L1). After double restriction enzyme digestion (EcoR I/Kpn I), the CD274 CDS was inserted into plasmid p3 $\times$ flag-CMV14. To obtain more effective expression, the KOZAK sequence was designed into primers, which were as follows:

\section{Forward primer, GGCC GAATTC GCCGCCACC ATGAGGATATTTGCTGTCTTTATATTC; Reverse primer, CTGA GGTACC TTACGTCTCC TCCAAATGTGTATCACTTTG.}

The EZH2 and IRF1 overexpression plasmids were purchased from VectorBuilder company (https://www. vectorbuilder.cn/).

\section{Chromatin immunoprecipitation ChIP}

ChIP was performed by using SimpleChIP ${ }^{\circ}$ Enzymatic Chromatin IP Kit (Cell Signaling Technology). Crosslinking was performed with $1 \%$ paraformaldehyde for 10 
min. Micrococcal nuclease was added to digest the DNA to lengths of approximately $150-900 \mathrm{bp}$. The digested DNA solution was sonicated using a Qsonica Q700 sonicator (Qsonica, Newtown, CT, USA) for $1 \mathrm{~min}$ at an amplitude of $15 \%$. Protein-DNA complexes were precipitated using specific antibodies against H3K27me3 (Cell Signaling Technology) and IgG control (Cell Signaling Technology). ChIP-enriched chromatin was used for RT-PCR with a SYBR green real-time PCR kit (Toyobo); the data were normalized to the input. The specific primers are listed in Additional file 2: Table S6.

\section{Prediction of CpG Islands on the CD274 promoter and bisulfite sequencing PCR}

The sequence of the human CD274 promoter was obtained from the EPD database (https://epd.vital-it.ch/ index.php). MethPrimer software (http://www.urogene. org/methprimer/) was used to predict CpG islands and design bisulfite-specific primers for amplification and sequencing. Only one $\mathrm{CpG}$ island was predicted on the CD274 promoter ( $-2000 \mathrm{bp}$ to $+500 \mathrm{bp}$ ). The bisulfitespecific primers sequences were as follows:

\section{Forward primer, ATTTGTTGTTTTGGGTAG AGGTG; \\ Reverse primer, TAACTCTACTACCCCCTA AACCATC.}

\section{Transcriptome profiling analysis}

In current study, we used the same batch of transcriptome profiling data previously used [32], with differences in grouping. According to the status of immune activation which was defined by the expression level of PD-L1 on the infiltrated M $\phi s$, the HCC tissues were divided into two groups: immune-activated and immunesuppressed [32] (12 cases in each group).

Gene with fold change (FC) more than two between groups and with a Student's t tests $p$ value $<0.05$ was defined as a differentially expressed gene (DEG). And the expression data of all DEGs were analyzed to form heatmap by Funrich 3.1.3 software. The genes upregulated in immune-activated group were further analyzed for GO term enrichment by Funrich 3.1.3 software.

\section{Statistical analysis}

Differences in the means for continuous variables were compared using Student's $t$ test or analysis of variance, and differences in the proportions were tested using the $\chi^{2}$ test. Kaplan-Meier estimates were calculated and compared using the log-rank test. Cox proportional hazard regression models were applied to evaluate the prognostic variables for OS and RFS. IBM SPSS (version 21.0; IBM Corp., Armonk, NY, USA) statistics software was used for all statistical analyses. All data were analyzed using two-tailed tests unless otherwise specified, and $P<0.05$ was considered statistically significant.

\section{Results \\ EZH2 negatively regulates the IFNY-induced PD-L1 expression}

To evaluate the potential role of EZH2 in regulating PDL1, we first investigated its expression in HCC tissues (Fig. 1a). IHC staining showed that EZH2 was highly expressed on hepatoma cells in HCC tumors compared with that on parenchyma cells in the non-tumor region. We noted that a few stroma cells were also positive for $\mathrm{EZH} 2$. Considering that $\mathrm{Mo} / \mathrm{M} \phi \mathrm{s}$ are the major PD-L1expressing stroma cells in HCC tumors, we performed multiplex staining to analyze EZH2 expression on $\mathrm{Mo} /$ M $\phi$ s. However, EHZ2 was weakly detected on $\mathrm{Mo} / \mathrm{M} \phi \mathrm{s}$ in HCC tumor tissues (Fig. 1b).

Recently, we observed that over $70 \%$ of $\mathrm{HCC}$ tissues were weak or negative for PD-L1 expression [32]. To evaluate the effect of EZH2 on PD-L1 expression, we used different hepatoma cell lines treated with IFN $\gamma$, which is a potent PD-L1 induction factor in multiple tumors [34, 35]. As shown in Fig. 1c, hepatoma cells expressed high level of EZH2, and had only marginally increased PD-L1 expression upon IFN $\gamma$ stimulation. In contrast, monocytes expressed a low level of EZH2, and showed significantly upregulated PD-L1 expression in response to IFN $\gamma$. We then performed RNAi assays to elucidate the regulatory effect of EZH2 on PD-L1. Immunoblotting assays showed that the effective EZH2targeted siRNAs enhanced IFNY-induced PD-L1 expression in hepatoma cell (Fig. 1d). Flow cytometry analyses showed that IFN $\gamma$-induced PD-L1 expression peaked at 18 to $24 \mathrm{~h}$ (Additional file 1: Figure S2) post stimulation. Furthermore, qPCR, immunoblotting, and flow cytometry analyses confirmed that IFN $\gamma$-induced PD-L1 expression was upregulated in a variety $E Z H 2$-silenced hepatoma cells (Fig. 1e-g). These data suggested that EZH2 could negatively regulate IFN $\gamma$-induced PD-L1 expression in hepatoma cells.

\section{EZH2-mediated H3K27me3 on the CD274 promoter to control PD-L1 expression}

EZH2-mediated H3K27me3 often leads to epigenetic silencing of target genes [36]; thus we first tested whether EZH2 could directly regulate the H3K27me3 levels on the CD274 (the gene encoding PD-L1) promoter to suppress IFN $\gamma$-induced PD-L1 expression. A concentration gradient of DZNep (an inhibitor of all Sadenosylmethionine (SAM)dependent enzymes, including EZH2) [37] and GSK126 (a selective inhibitor of EZH2 methyltransferase activity) [38] were applied to reprogram the epigenetic pathways in hepatoma cells. As expected, GSK126 significantly inhibited the level of 

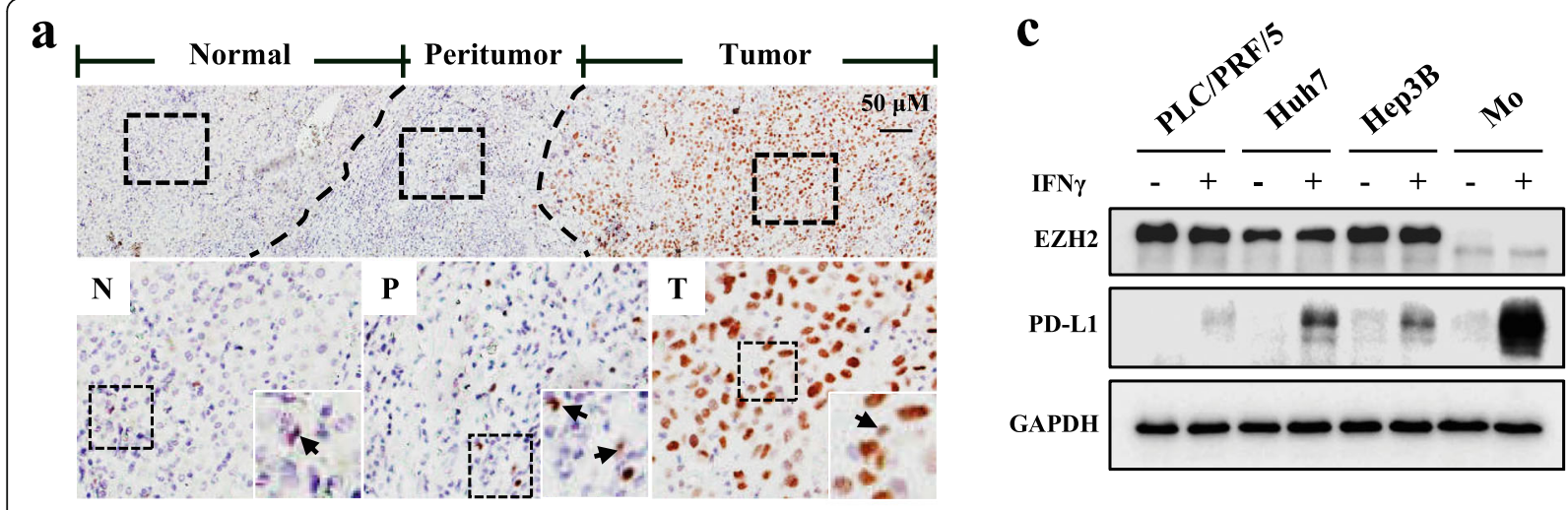

b
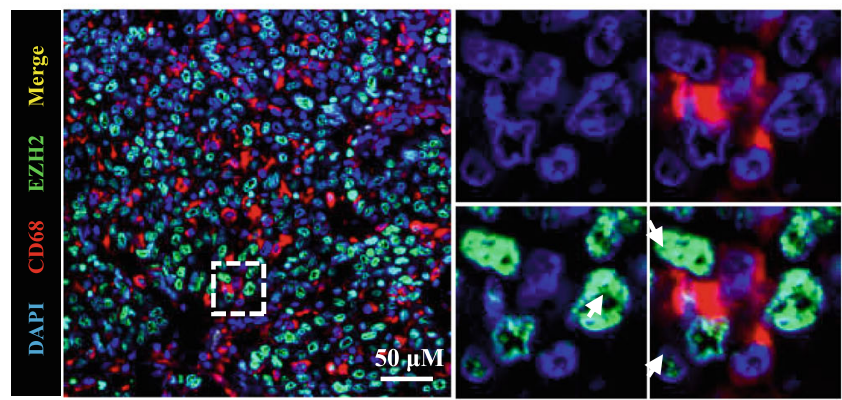

d

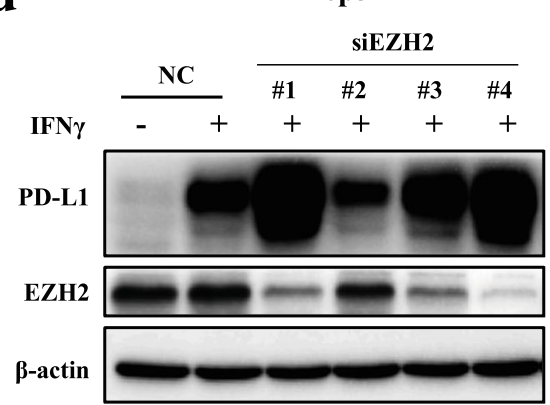

$\mathbf{e}$

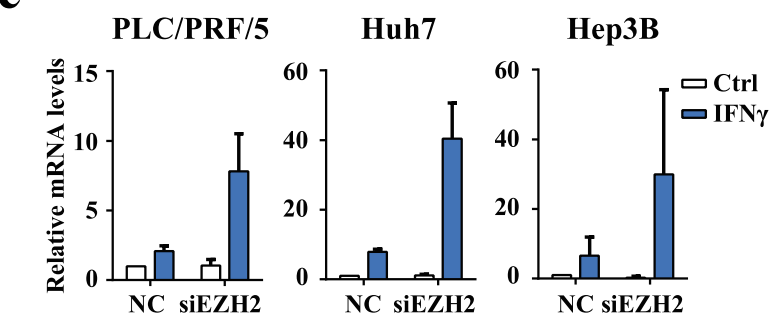

f

PLC/PRF/5 Huh7 Hep3B

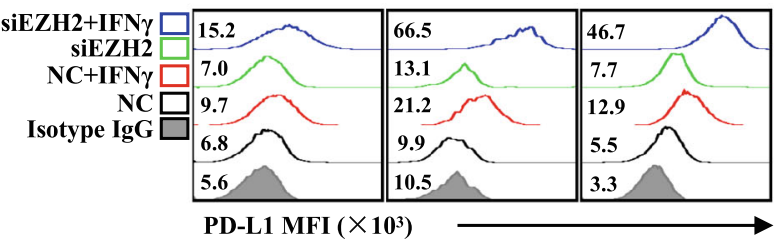

g

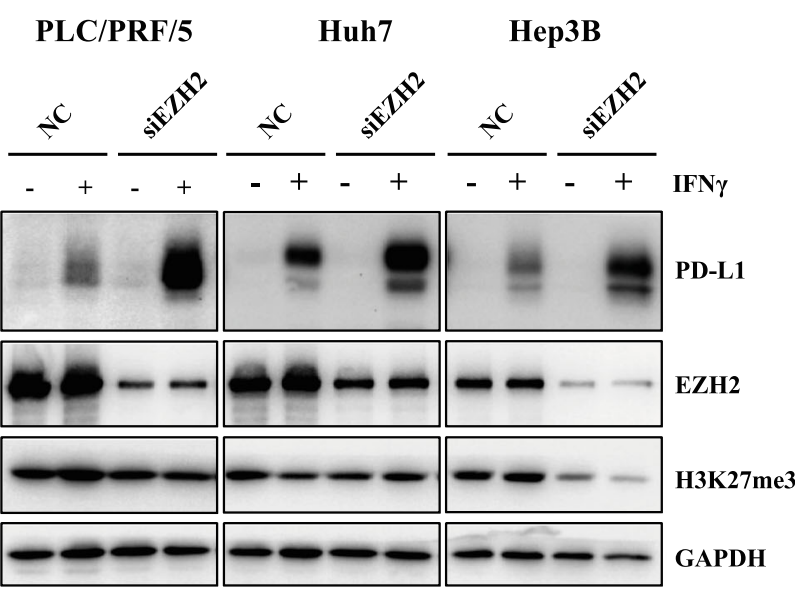

Fig. 1 EZH2 negatively regulates the IFNY-induced PD-L1 expression. a Representative IHC staining of EZH2 in HCC tissues. The black arrows indicate the expression of EZH2 on stroma cells, and the red arrows indicate the expression of EZH2 on TCs. $\mathbf{b}$ Representative pictures of multiple immunofluorescence staining showing the expression of EZH2 (green) and CD68 (red) in HCC. Scale bar, $50 \mu m$. The white arrows indicate Mo/ $M \varphi s$, and the five-pointed stars indicate TCs. c Immunoblotting analysis showing the expression of IFNY-induced PD-L1 in hepatoma cells and monocytes. $\mathbf{d}$ Hep3B cells were transfected with negative control (NC) or different EZH2-targeted siRNAs for $48 \mathrm{~h}$, and then treated with IFNY for $24 \mathrm{~h}$. Immunoblotting analyses were performed to detect the expression of EZH2 and PD-L1. $\beta$-actin was used as a loading control. e qPCR analysis showing that downregulation of EZH2 promoted the mRNA expression of IFNy-induced PD-L1 in PLC/PRF/5, Huh7, and Hep3B cells. $\mathbf{f}$ FACS staining showing that downregulation of EZH2 promoted the expression of IFNY-induced PD-L1 in PLC/PRF/5, Huh7, and Hep3B cells. g Downregulation of EZH2 increased the protein level of IFNY-induced PD-L1 in PLC/PRF/5, Huh7, and Hep3B cells. The indicated hepatoma cells were transfected with EZH2-targeted or NC siRNA for $48 \mathrm{~h}$, and then treated with IFNY for an additional $24 \mathrm{~h}$. Immunoblotting analysis was performed to detect the protein levels of PD-L1, EZH2, and H3K27me3. GAPDH was used as a loading control 
H3K27me3 without affecting EZH2 expression, while DZNep simultaneously downregulated the expression of EZH2 (Additional file 1: Figure S3a-b). Inhibiting EZH2 by GSK126 or DZNep treatment effectively increased IFN $\gamma$-induced PD-L1 expression in hepatoma cells (Fig. 2a, Additional file 1: Figure S3c-e). ChIP-qPCR analysis revealed that $\mathrm{H} 3 \mathrm{~K} 27 \mathrm{me} 3$ occupancy on the promoter of CD274 was significantly downregulated in Hep3B-shEZH2 cells compared with that of the control cells (Fig. 2b). These data suggested that H3K27me3 modification at the promoter level is involved in EZH2mediated PD-L1 repression.

It has been reported that EZH2 could also serve as a recruitment platform for the DNA methyltransferase, DNMT1 [39]. From the MethPrimer online database, we predicted $\mathrm{CpG}$ islands that are enriched near the transcription start site of CD274 [40] (Fig. 2c). However, these CpG islands are barely methylated in these hepatoma cell lines, as analyzed by bisulfite sequencing PCR (BSP) (Fig. 2d). Furthermore, The Cancer Genome Atlas (TCGA) data showed no correlation between mRNA and promoter methylation levels of CD274 in $\mathrm{HCC}$ tumor tissues (Fig. 2e).

It has been reported that the upregulated expression of key molecules such as, interferon gamma receptor 1 (IFNGR1), IFNGR2, Janus kinase 1 (JAK1), and JAK2, activates the IFN $\gamma$-STAT1 signaling in response to IFN $\gamma$ stimulation. We evaluated whether their expression, and the activation of IFN $\gamma$-STAT1 signaling, were influenced by EZH2. The results showed that the mRNA levels of these molecules and STAT1 phosphorylation (Fig. 2f-g) were not affected by $E Z H 2$ silencing (Additional file 1: Figure S4a-b).

Taken together, these data demonstrated that EZH2 directly regulates the $\mathrm{H} 3 \mathrm{~K} 27 \mathrm{me} 3$ levels, but not DNA methylation, of the CD274 promoter. Moreover, activation of IFN $\gamma$-STAT1 signaling is not influenced by EZH2 expression in hepatoma cells.

\section{EZH2 inhibits PD-L1 expression by epigenetic silencing of IRF1 expression}

To further explore the regulatory effect of EZH2 on the CD274 promoter, we constructed a promoter luciferase reporter plasmid containing different truncated versions of the CD274 promoter without H3K27me3 modification (Fig. 3a) and examined the transcription activity of these truncated promoters using dual-luciferase reporter assays. As shown in Fig. 3b, all the designed promoters exhibited similarly enhanced luciferase activity in EZH2silenced cells when treated with IFNy, indicating that EZH2 might regulate CD274 transcription by affecting the activities of certain transcription factors (TF) that bind to the P1 truncated promoter. Thirty-nine TFs were predicted to bind to the $\mathrm{P} 1$ promoter on the
PROMO website [41, 42] and 469 genes who showed co-expression with $C D 274$ with correlation coefficients more than 0.3 were screened out from the cBioportal website (HCC, TCGA, Provisional) [43, 44]. The Venn diagram analysis identified IRF1 as the only potential candidate gene that met both screening criteria (Fig. 3c-d, Additional file 3: Table S7).

Next, dual-luciferase reporter assays were performed to examine the effect of IRF1 on the transcriptional activity of CD274 promoter. The results showed that the luciferase activity of P1 promoter was enhanced by knockdown of EZH2, and this enhancement could be impaired by IRF1 silencing (Fig. 3e). Consistent with this, ectopic expression of IRF1 partially restored the luciferase activity of $\mathrm{P} 1$ promoter reduced by overexpression of EZH2 (Fig. 3f). Moreover, re-expression of IRF1 also partly rescued the reduced luciferase activity of P1 caused by knockdown of IRF1 in the EZH2silenced hepatoma cells (Fig. 3g). We predicted two IRF1 response elements (IREs) on the P1 truncated promoter of CD274 using the JASPAR database (Fig. 3h). To investigate the activities of these response elements, we constructed P1 truncated promoter luciferase reporter plasmids that were deleted for IRE1, IRE2, or both, which were named as $\triangle \mathrm{IRE} 1, \triangle \mathrm{IRE} 2$, and $\triangle \mathrm{IREs}$, respectively (Fig. 3i). Luciferase assays identified that the transcriptional activity of $\triangle \mathrm{IRE} 1, \triangle \mathrm{IRE} 2$, and $\triangle$ IREs were weakened as compared with wild-type P1 in Huh7 and Hep3B cells after stimulation with IFN $\gamma$ (Fig. 3j). These data indicated that the transcriptional regulation of CD274 by EZH2 is also influenced by the level of IRF1.

Based on the above results, we speculated that IRF1 is downstream of EZH2 to involve in PD-L1 regulation. To examine whether IRF1 is upregulated prior to PD-L1 in EZH2-silencd cells under IFNy treatment, we evaluated the expression dynamics of IRF1 and PD-L1. As expected, IRF1 was induced and reached its maximum level prior to that of PD-L1 (Fig. 4a, Additional file 1: Figure S5a-b). Moreover, knockdown of IRF1 in EZH2silenced hepatoma cells decreased the abundance of PDL1 (Fig. 4b). These results suggested IRF1 should be involved in the EZH2-regulated expression of PD-L1.

Of note, we observed that the expression of IRF1 and PD-L1 were significantly reduced after the reexpression of EZH2 in the EZH2-silenced cells; however, PD-L1 expression was only slightly increased after further ectopic expression of IRF1 (Fig. 4c, Additional file 1: Figure S5c). Considering the epigenetic silencing of EZH2 on PD-L1, we speculated IRF1 has limited induction effect on PD-L1 expression in cells with high expression of EZH2. To further verify this, the induction effect of IRF1 on PD-L1 expression was evaluated in EZH2-silenced hepatoma cells. The results showed that knockdown of IRF1 significantly 
a

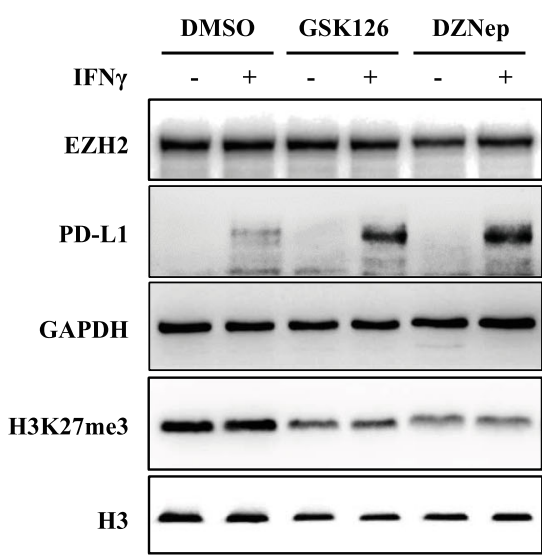

c

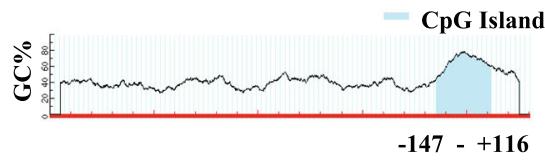

e

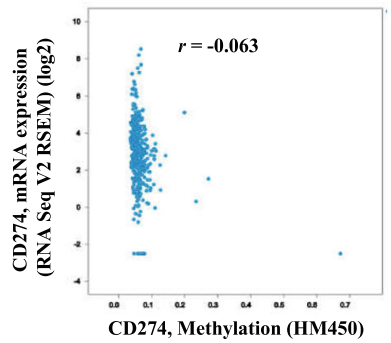

f

Huh7

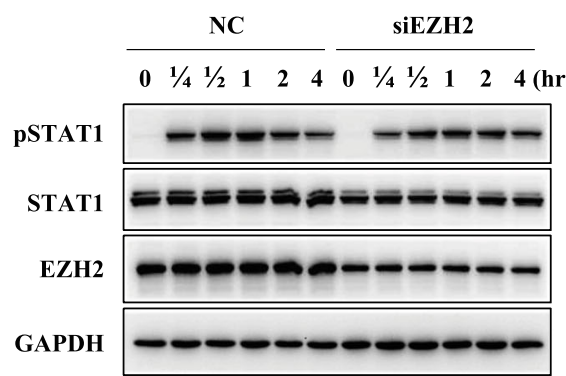

b

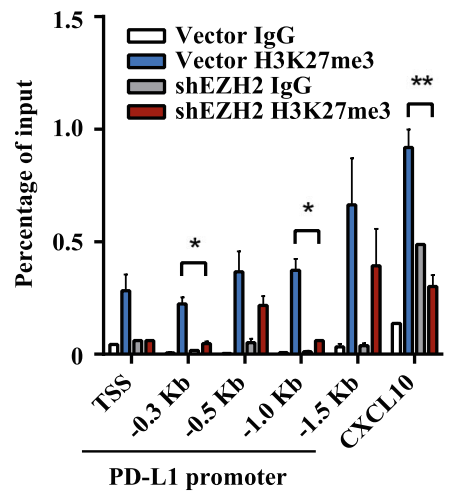

d<smiles>[CH][CH]</smiles>
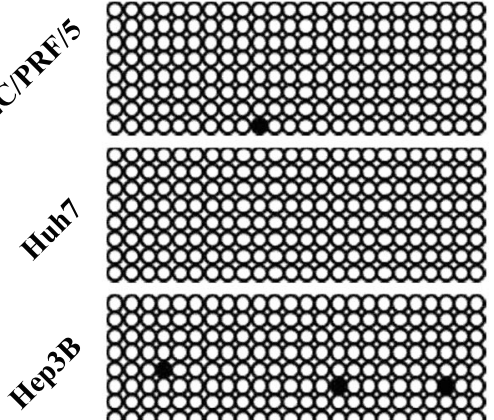

g

\section{PLC/PRF/5}

NC

siEZH2

$\begin{array}{lllllllllllll}\text { IFN } \gamma & 0 & 1 / 4 & 1 / 2 & 1 & 2 & 4 & 0 & 1 / 4 & 1 / 2 & 1 & 2 & 4 \\ (h r)\end{array}$

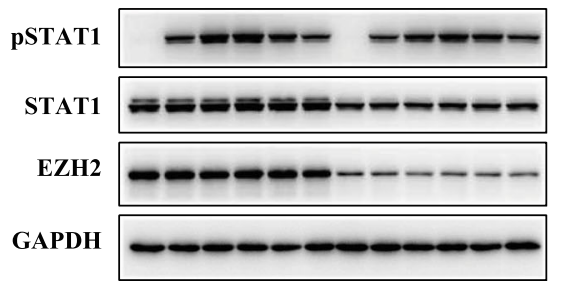

Fig. 2 EZH2-mediated H3K27me3 on the CD274 promoter controls PD-L1 expression. a Hep3B cells were pretreated with GSK126, DZNep, or DMSO for $48 \mathrm{~h}$, and then treated with IFNy for an additional $24 \mathrm{~h}$. Immunoblotting was performed to detect the protein expression of PD-L1, $\mathrm{EZH} 2$, and H3K27me3. GAPDH and H3 were used as loading controls. b An H3K27me3 ChIP assay was performed in shEZH2 Hep3B and vector control cells. H3K27me3 levels at the CD274 (PD-L1) promoter were normalized to the input. TSS, transcription start site, $-0.3,-0.5,-1.0$, and $1.5 \mathrm{~kb}$ indicate the corresponding upstream loci of the CD274 gene TSS. CXCL10 was used as a positive control. (Mean \pm S.E.M.; $n=3$; $* P<0.05$, ** $P<0.01$, Wilcoxon test). c Diagram of the CpG island distribution on $-2000 \mathrm{nt}$ to $+250 \mathrm{nt}$ region of the CD274 promoter predicted by MethPrimer website. $\mathbf{d}$ DNA methylation on the CD274 promoter. DNA methylation at CpG sites was quantified using bisulfite sequencing. Filled circle, methylated; open circle, unmethylated. e DNA methylation and gene expression data for PD-L1 from TCGA HCC tissues were analyzed on the cBioportal website. The Pearson correlation coefficient $(r)$ is shown. $\mathbf{f}$ and $\mathbf{g}$ Effect of downregulation of EZH2 on the IFNy-STAT1 signaling activation. Huh7 (f) or PLC/PRF/5 (g) cells were pre-transfected with EZH2-targeted siRNA or NC for $48 \mathrm{~h}$, and then treated with IFNy for 0-4 h. Immunoblotting was performed to detect the levels of pSTAT1 and EZH2. STAT1 and GAPDH were used as loading controls for pSTAT1 and EZH2 respectively

reduced the expression of PD-L1, and this reduction was rescued by re-expression of $I R F 1$ (Fig. 4d, Additional file 1: Figure S5d).
We also analyzed the impact of EZH2/H3K27me3 axis inhibitors on IRF1 expression. The results showed that GSK126 and DZNep treatments promoted IFNy-induced 
a

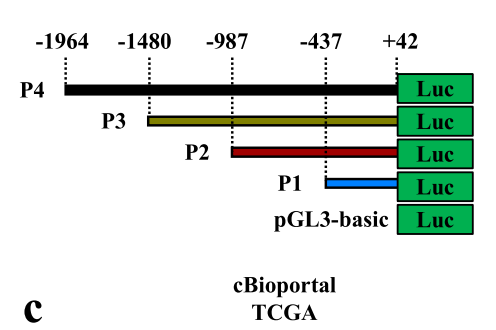

b

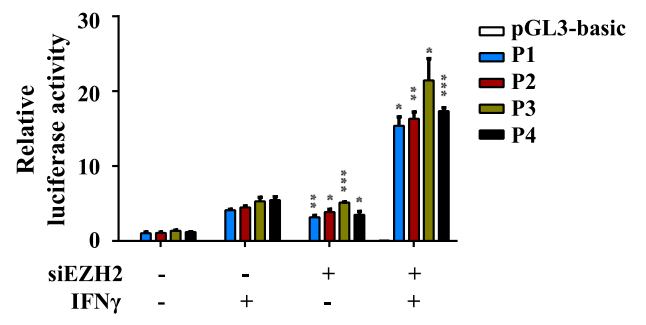

d

PROMO

38

IRF-1

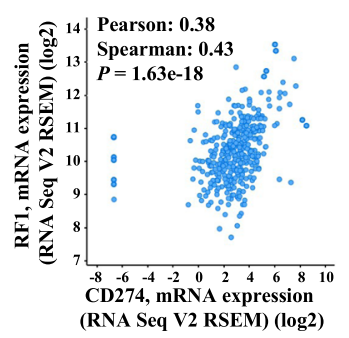

f

Huh7 Hep3B

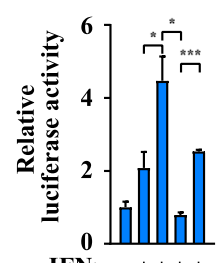

IFN $\gamma_{-}++++$ SiEZH2 - - +++ pEZH2 - - + + pIRF-1 - - +

P1 + pRL-TK $+++++\quad+++++$

g

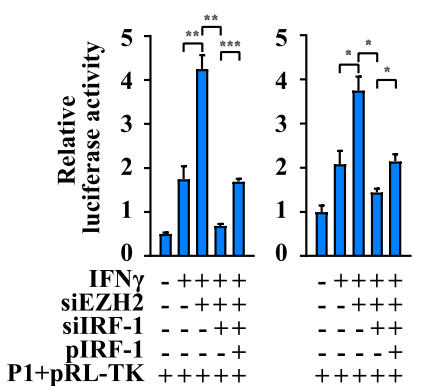

e

Huh7 Hep3B

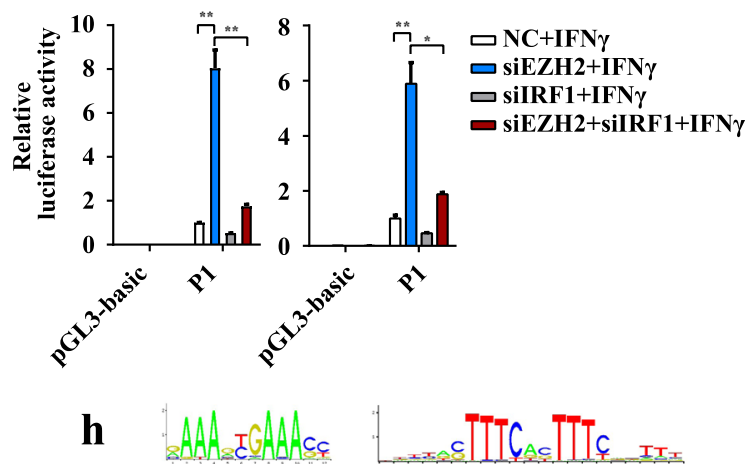

i

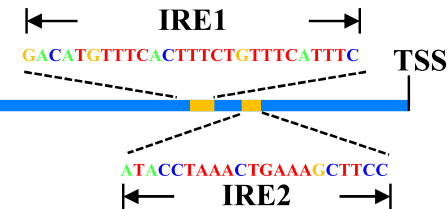

j

Huh7

Hep3B

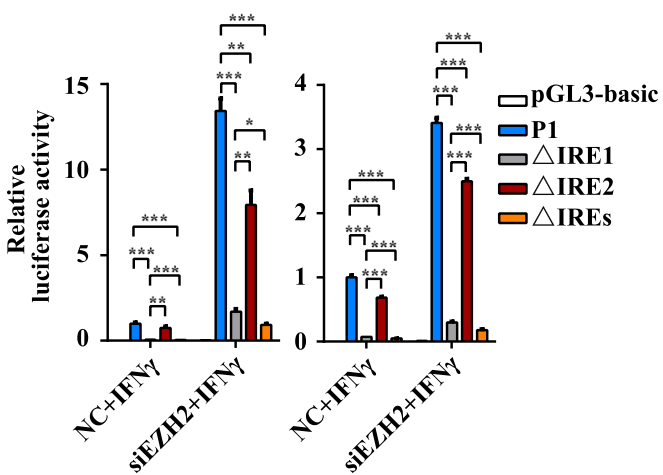

Fig. 3 (See legend on next page.) 
(See figure on previous page.)

Fig. 3 IRF1 is a potential transcription factor involved in the negative regulation of PD-L1 by EZH2. a Schematic diagram of a series of CD274 (PDL1) gene promoter luciferase reporter plasmids. b After transfection with EZH2-targeted or NC siRNA overnight, Huh7 cells were co-transfected with pGL3-basic vector or the indicated CD274 promoter luciferase reporter gene plasmid and the pRL-TK plasmid for $48 \mathrm{~h}$, and then treated with IFNy for an additional 24-h. Luciferase activity was determined and normalized using the dual luciferase reporter system. (Mean \pm S.E.M.; $n=3$; the asterisk represents a comparison between the siEZH2 group and the corresponding control group; ${ }^{*} P<0.05,{ }^{* *} P<0.01$, ${ }^{* * *} P<0.001$, Wilcoxon test). c TFs that could potentially bind to the P1 truncated promoter were predicted using the PROMO bioinformatics software (pink circle). Genes showing the absolute values of both the Pearson and Spearman expression correlation coefficient (positively or negatively correlated) of more than 0.3 with CD274 (PD-L1 gene) in HCC tissues (TCGA, Provisional) were analyzed on the cBioportal website (blue circle). Venn diagram showing that IRF-1 was the only candidate gene in both gene sets. d Scatter gram showing the mRNA expression correlation of CD274 and IRF1 from TCGA (HCC, Provisional). Pearson and Spearman correlation coefficients and $P$ values are shown. e Huh7 and Hep3B cells were transfected with NC or EZH2-targeted, IRF1-targeted, or both, siRNA overnight, and then co-transfected with pGL3-basic vector or the P1 luciferase reporter gene plasmid and pRL-TK plasmid for $48 \mathrm{~h}$. The cells were then treated with IFNy for an additional $24 \mathrm{~h}$. Luciferase activity was determined and normalized using the dual luciferase reporter system (Mean \pm S.E.M.; $n=4 ;{ }^{*} P<0.05,{ }^{*} P<0.01$, Wilcoxon test). f After transfection with NC or EZH2 siRNA targeting 3'-UTR, Huh7 and Hep3B cells were transfected with the indicated plasmids for $48 \mathrm{~h}$, and then treated with IFNy for $24 \mathrm{~h}$. Luciferase activity was determined and normalized using the dual luciferase reporter system (Mean \pm S.E.M.; $n=3 ;{ }^{*} P<0.05$, ** $P<0.01$, Wilcoxon test). pEZH2 and pIRF-1 represent ectopic expression of EZH2 and IRF-1 respectively, and the corresponding control groups were transfected with NC siRNA and/or vector plasmids. g After transfection with NC or the indicated siRNA targeting 3'-UTR, Huh7 and Hep3B cells were transfected with the indicated plasmids for $48 \mathrm{~h}$, and then treated with IFNy for $24 \mathrm{~h}$. Luciferase activity was determined and normalized using the dual luciferase reporter system (Mean \pm S.E.M.; $n=3{ }^{*} P<0.05,{ }^{* *} P<0.01$, Wilcoxon test). plRF-1 represent ectopic expression of IRF-1, and the corresponding control groups were transfected with NC siRNA and/or vector plasmids. $\mathbf{h}$ Sequence logo of IRF1 binding site frequency matrix of Homo sapiens predicted using the online software JASPAR. i Schematic representation of IRF1 binding sites in the CD274 P1 promoter region, as predicted by JASPAR. IRE, IRF1 response element. $\mathbf{j}$ Huh7 and Hep3B cells were transfected with NC or EZH2-targeted siRNA overnight, and then co-transfected with pGL3-basic vector or the indicated P1 with or without IREs sequence deletion luciferase reporter gene plasmid and pRL-TK plasmid for $48 \mathrm{~h}$. The cells were then treated with IFNy for an additional $24 \mathrm{~h}$. Luciferase activity was determined and normalized using the dual luciferase reporter system (Mean \pm S.E.M.; $n=4$; NS, no significant difference; ${ }^{*} P<0.05,{ }^{* *} P<0.01,{ }^{* * *} P<0.001$, Wilcoxon test)

IRF1 expression (Fig. 4e, Additional file 1: Figure S5e). ChIP-qPCR analysis was then performed to reveal whether EZH2 could suppress the expression of IRF1 through epigenetic machinery. The results showed H3K27me3 occupancy on the IRF1 promoter was significantly downregulated in Hep3B-shEZH2 cells compared with that of the control (Fig. 4f). These results suggested that IRF1 expression was suppressed by the EZH2H3K27me3 axis, which leads to inhibition of PD-L1 expression.

Taken together, these results demonstrated that IRF1 promotes the expression of PD-L1, which depends on the epigenetic modification levels of PD-L1 driven by EZH2.

\section{Correlation between EZH2 and PD-L1 expression in HCC tissues}

The above findings indicated the effect of EZH2 in regulating IFN $\gamma$-induced PD-L1 expression. Next, we analyzed the relationship between EZH2 and PD-L1 expression in HCC tumors. Our recent study showed that M $\phi-P D-L 1$ expression was related to the activated tumor microenvironment [32]. Transcriptome profiling and gene ontology biological analysis confirmed that $\mathrm{M} \phi-\mathrm{PD}-\mathrm{L}^{+} \mathrm{HCC}$ tumor samples displayed an immune-activated microenvironment and upregulated genes that were mainly involved in the IFN $\gamma$-mediated signaling pathway (Additional file 1: Figure S6a and b, Additional file 4: Table S8). Considering that IFN $\gamma$ stimulation was demonstrated to be required for EZH2-mediated PD-L1 expression in the cell experiments, we analyzed the association between EZH2 and PD-L1 in samples with different microenvironments. Statistical analyses showed a significantly negative correlation between PD-L1 and EZH2 levels on TCs in immune-activated HCC tissues, but not in the total or immune-suppressed samples (Fig. 5a). Multiple immunofluorescence staining revealed that EZH2 protein was barely detected on either PD-L1 ${ }^{+} \mathrm{M} \phi s$ or TCs (Fig. 5b). Collectively, these results suggested that EZH2 was negatively correlated with PD-L1 expression in the immune-activated HCC tumor microenvironment.

Survival analysis showed that the high levels of EZH2 in hepatoma cells indicated poor OS $(P=0.013)$ and RFS $(P=0.002)$ in HCC patients (Fig. 5c, f). Moreover, patients with low or high levels of EZH2 could be further divided into two groups by their PD-L1 expression on TCs. Kaplan-Meier curves showed that patients with PDL1 expression on TCs had poor OS and RFS (Fig. 5d, OS $P=0.025$; Fig. 5g, RFS $P=0.020)$ in EZH2 low-expression group but not in EZH2 high-expression group (Fig. 5e, OS $P=0.072$; Fig. 5h, RFS $P=0.230$ ). Multivariate Cox analysis demonstrated that the combination of EZH2 and PD-L1 was an independent prognostic factor for both OS $(P=0.01)$ and RFS $(P=0.001)$ in HCC patients (Table 1$)$.

\section{Discussion}

Accumulating evidence indicates that epigenetic inhibitors could improve the therapeutic efficacy of immune checkpoint blockade [21, 45]; however, the exact regulatory mechanism is not fully understood. In the present 
$\mathbf{a}$

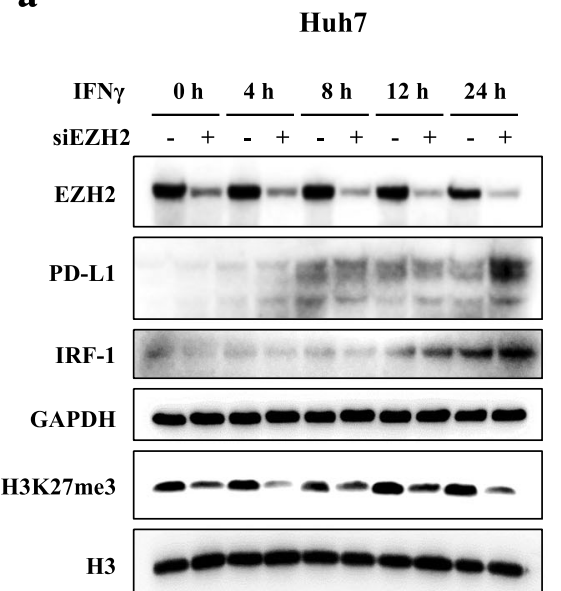

b

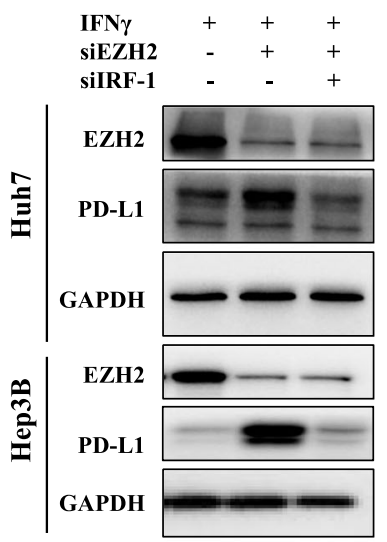

e

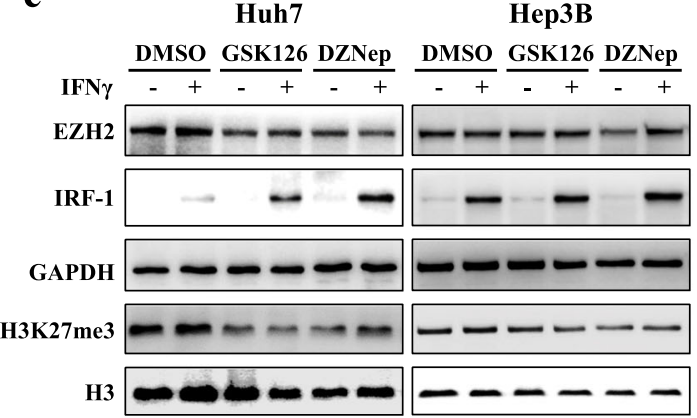

c

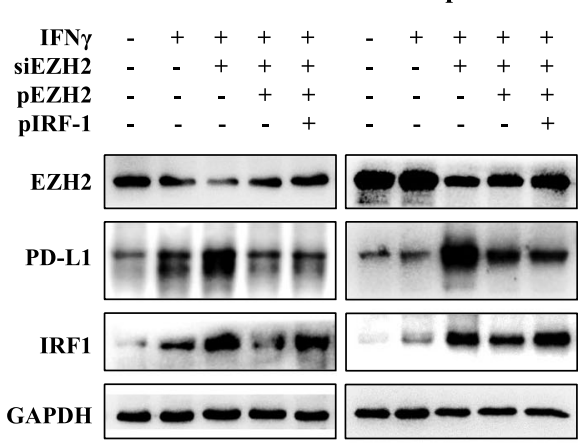

d

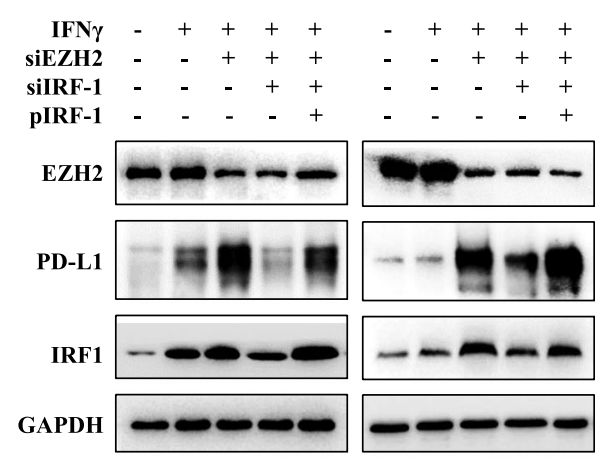

f

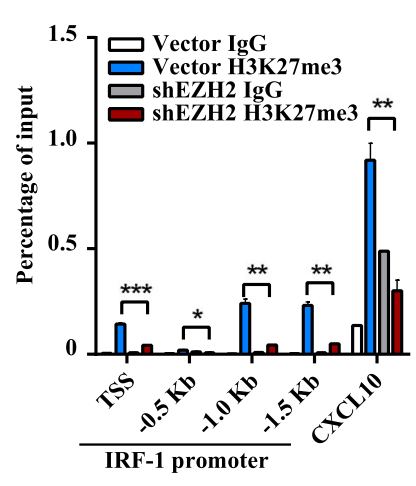

Fig. 4 EZH2 inhibits PD-L1 transcription by inhibiting transcription factor IRF1. a Huh7 cells were transfected with NC or EZH2-targeted siRNAs for $72 \mathrm{~h}$, and then treated with IFNy for the indicated times. Immunoblotting analysis was performed to detect the levels of EZH2, IRF1, and PD-L1. GAPDH was used as a loading control. b Huh7 and Hep3B cells were transfected with NC or EZH2-targeted siRNAs, with or without IRF1-targeted siRNAs for $48 \mathrm{~h}$, and then treated with IFNy for $24 \mathrm{~h}$. Immunoblotting was performed to detect the levels of EZH2 and PD-L1. c After transfection with EZH2 siRNA targeting 3'-UTR, Huh7 and Hep3B cells were transfected with the indicated plasmids for $48 \mathrm{~h}$, and then treated with IFNY for 24 h. Immunoblotting was performed to detect the levels of EZH2, IRF-1 and PD-L1. d After transfection with the indicated siRNA targeting 3'-UTR, Huh7 and Hep3B cells were transfected with the indicated plasmids for $48 \mathrm{~h}$, and then treated with IFNy for $24 \mathrm{~h}$. Immunoblotting was performed to detect the levels of EZH2, IRF-1 and PD-L1. In $\mathbf{c}$ and $\mathbf{d}$, the corresponding control groups were transfected with NC siRNA or vector plasmids. pEZH2 and plRF-1 represent ectopic expression of EZH2 and IRF-1, respectively. e Huh7 and PLC/PRF/5 cells were pretreated with GSK126, DZNep, or DMSO for $48 \mathrm{~h}$, and then treated with IFNy for an additional $12 \mathrm{~h}$. GAPDH and $\mathrm{H} 3$ were used as loading controls for EZH2 and H3K27me3, respectively. f An H3K27me3 ChIP assay was performed in shEZH2 Hep3B and vector control cells. H3K27me3 levels on the IRF1 gene promoter were normalized to the input. TSS, transcription start site; $-0.5 \mathrm{~kb},-1.0 \mathrm{~kb},-1.5 \mathrm{~kb}$ indicate the corresponding upstream locus in the IRF1 gene TSS. CXCL10 was used as positive control (Mean \pm S.E.M.; $n=3$; ${ }^{*} P<0.05$, ** $P<0.01$, *** $P<0.001$, Wilcoxon test) 


\section{a}

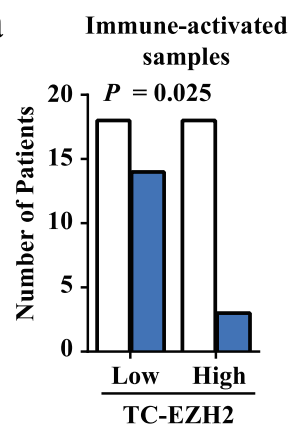

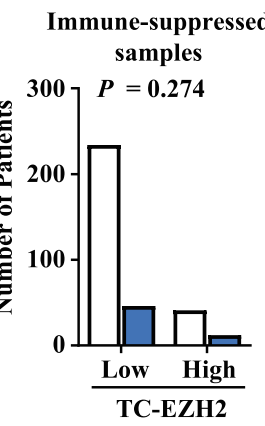

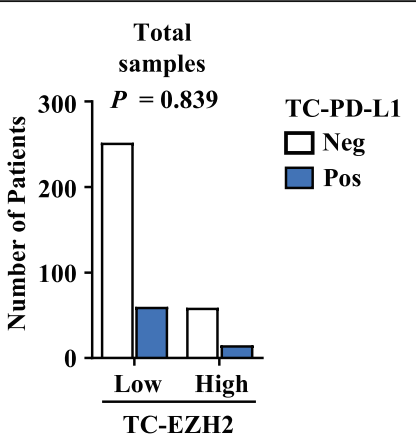

b
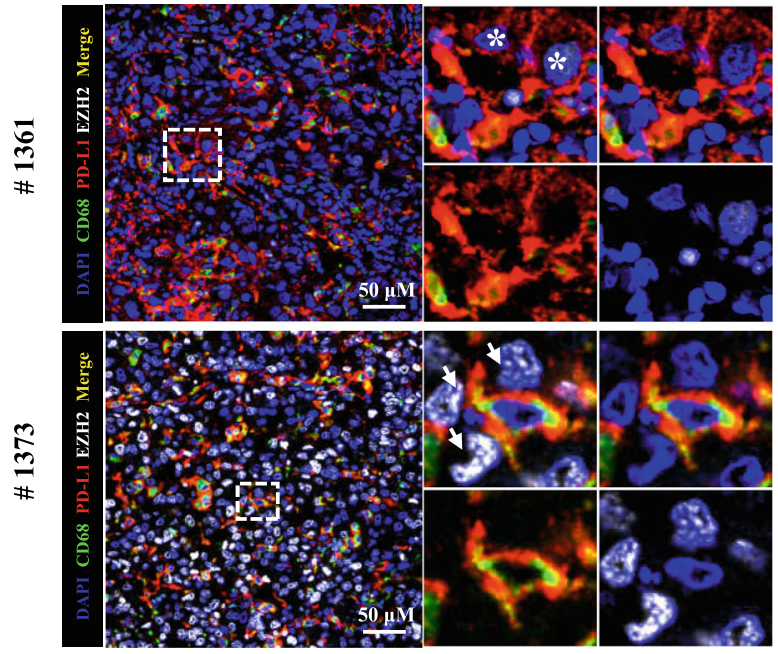

C

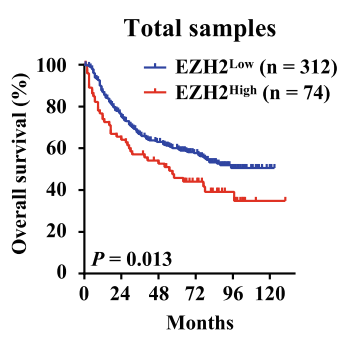

d

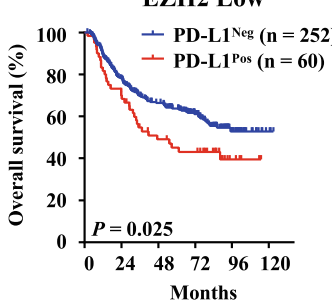

e

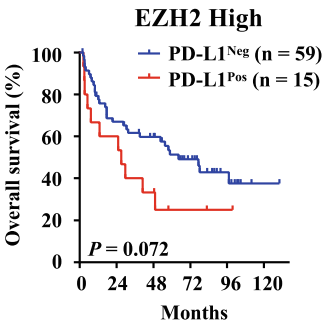

f

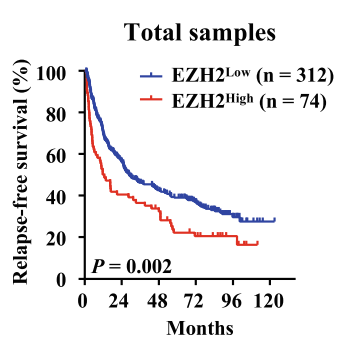

g

EZH2 Low

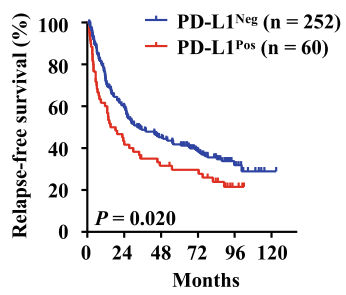

h

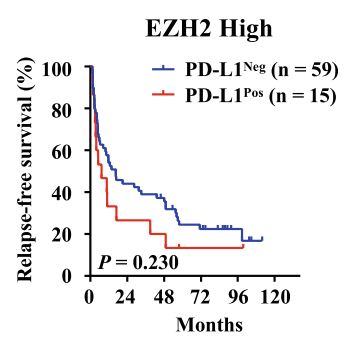

Fig. 5 Correlation between EZH2 and PD-L1 expression in HCC tissues. a The expression correlation statistics of EZH2 and PD-L1 on hepatoma cells in immune-activated (left), immune-suppressed (middle), or all ungrouped (right) HCC tissues. b Representative images of multiple immunofluorescence staining showing the expression of EZH2 (gray) and PD-L1 (red) in HCC. Scale bar, $50 \mu$ m. \#1361 and \#1373 indicates the ID number of the HCC samples. The white stars and arrows indicate tumor cells expressing low or high levels of EZH2, respectively, and the fivepointed stars indicate the PD-L1 ${ }^{+}$Mo/M $\varphi$ s with EZH2 low-expression. c Cumulative OS of EZH2 was calculated using the Kaplan-Meier method and analyzed using the log-rank test $(P=0.013)$. Cumulative OS of PD-L1 was calculated using the Kaplan-Meier method and analyzed using the log-rank test in patients with EZH2 low expression $(\mathbf{d} P=0.025)$ and high expression $(\mathbf{e} P=0.072)$. f Cumulative OS of EZH2 combined with PD-L1 was calculated using the Kaplan-Meier method and analyzed using the log-rank test $(P=0.002)$. Cumulative RFS of PD-L1 was calculated using the Kaplan-Meier method and analyzed using the log-rank test in patients with EZH2 low expression $(\mathbf{g} P=0.020)$ and high expression (h $P=$ 0.230). $\left.{ }^{*} P<0.05, * * * P<0.001\right)$ 
Table 1 Univariate and multivariate analysis of factors associated with overall survival and relapse-free survival

\begin{tabular}{|c|c|c|c|c|c|c|c|c|}
\hline \multirow[t]{3}{*}{ Variables } & \multicolumn{4}{|l|}{ OS } & \multicolumn{4}{|l|}{ RFS } \\
\hline & \multirow{2}{*}{$\begin{array}{l}\text { Univariate } \\
p\end{array}$} & \multicolumn{3}{|c|}{ Multivariate } & \multirow{2}{*}{$\begin{array}{l}\text { Univariate } \\
p\end{array}$} & \multicolumn{3}{|c|}{ Multivariate } \\
\hline & & $\mathrm{HR}$ & $95 \% \mathrm{Cl}$ & $p$ & & $\mathrm{HR}$ & $95 \% \mathrm{Cl}$ & $p$ \\
\hline Gender (female/male) & 0.396 & & & & 0.528 & & & \\
\hline Age,y $(>50 / \leq 50)$ & 0.711 & & & & 0.346 & & & \\
\hline HBsAg (present/absent) & 0.759 & & & & 0.107 & & & \\
\hline AFP, ng/ml (> 25/ $\leq 25)$ & $<0.0001$ & 1.588 & $1.122-2.249$ & 0.009 & 0.001 & 1.258 & $0.963-1.643$ & 0.092 \\
\hline 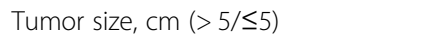 & 0.011 & 0.905 & $0.645-1.271$ & 0.565 & 0.040 & 0.928 & $0.706-1.219$ & 0.590 \\
\hline Tumor multiplicity (multiple/solitary) & $<0.0001$ & 1.468 & $1.006-2.142$ & 0.046 & $<0.0001$ & 1.518 & $1.094-2.107$ & 0.012 \\
\hline Vascular invasion (present/absent) & $<0.0001$ & 3.389 & $2.205-5.209$ & $<0.0001$ & $<0.0001$ & 2.257 & $1.547-3.293$ & $<0.0001$ \\
\hline TNM stage $(I I I+I V / I+I I)$ & $<0.0001$ & 1.632 & $1.086-2.453$ & 0.018 & $<0.0001$ & 1.294 & $0.914-1.831$ & 0.146 \\
\hline Differentiation (III + IV/I + II) & 0.55 & & & & 0.700 & & & \\
\hline Combination of EZH2 and PD-L1 & 0.0004 & 1.228 & $1.050-1.436$ & 0.010 & 0.0002 & 1.254 & $1.100-1.430$ & 0.001 \\
\hline
\end{tabular}

Variables associated with overall survival or relapse-free survival by univariate analysis were adopted as covariates in multivariate analysis and entered into the equation by the forward selection based on likelihood ratio test

Abbreviations: OS Overall survival, RFS Relapse-free survival, HR Hazard ratio, Cl Confidence interval

study, we reported that the epigenetic modifier EZH2 negatively regulated IFN $\gamma$-induced PD-L1 expression in hepatoma cells. Mechanistic studies demonstrated that EZH2 could suppress PD-L1 expression by upregulating the promoter H3K27me3 levels of CD274 (encoding PDL1) and IRF1 without affecting activation of the IFN $\gamma$ STAT1 pathway. A negative correlation between EZH2 and PD-L1 expression on TCs was demonstrated in HCC tissues with an immune-activated microenvironment. Moreover, the combination of EZH2 and PD-L1 on TCs was an independent prognostic factor for OS and RFS in patients with HCC.

Our previous studies demonstrated the differentiated expression of PD-L1 on TCs and Mo/M $\phi$ s had opposite clinical impacts on HCC patients [32]. Transcriptome profiling analysis showed that the immune-activated microenvironment was associated with PD-L1 expression on $\mathrm{Mo} / \mathrm{M} \phi \mathrm{s}$ but not $\mathrm{TCs}$, indicating that the immune-induced PD-L1 expression by TCs might be regulated by certain intrinsic factors. In the present study, we found that hepatoma cells expressed high levels of EZH2, which abrogated PD-L1 upregulation by IFN $\gamma$. In contrast, Mo/M $\phi$ s had low levels of EZH2 in HCC tumors and showed significantly upregulated PD$\mathrm{L} 1$ expression after IFN $\gamma$ stimulation. It should be noted that the criteria for evaluation of $\mathrm{EZH} 2$ positive expression were ambiguous in different studies [46, 47]. We determined the expression of EZH2 by nuclear expression on tumor cells, and quantified by $\mathrm{H}$-score. The cutoff value was chosen by the minimum $P$ value that divided patients with diverse clinical outcomes, and also highlighted the correlation between EZH2 and PD-L1 expression. The biological and clinical significance of this cutoff value should be further validated in other cohorts. Taken together, our data revealed that EZH2 acts as an intrinsic modifier that could influence PD-L1 expression in hepatoma cells.

Our mechanistic studies showed that EZH2 epigenetically silenced IFN $\gamma$-induced PD-L1 expression by upregulation of the H3K27me3 levels on the promoters of both CD274 and IRF1. Notably, EZH2 did not affect the activation of IFN $\gamma$-STAT1 signaling in hepatoma cells, as analyzed by qPCR and immunoblotting assays. This is different from other tumor models, such as in mycdriven prostate cancer, in which $E Z H 2$ knockdown restored IFNGR1 expression and further led to activation of IFN-JAK-STAT1 signaling [48]. Recently, it has been reported that the expressions of $\mathrm{EZH} 2$ and $\mathrm{PD}-\mathrm{L} 1$ were positively correlated in lung adenocarcinoma, and DNA methylation could be involved in regulating PD-L1 expression $[49,50]$. However, our BSP analysis in hepatoma cells with low constitutive expression of PD-L1 showed almost no methylation on the CD274 promoter regions that were predicted to be CpG islands (Fig. 2d). These data indicated that the intrinsic modifiers and extrinsic inducers for PD-L1 could be varied in different tumor models, for instance, the inflamed liver microenvironments due to chronic viral infection could have great impact on PD-L1 expression.

EZH2 inhibitor intervention experiments showed that both GSK126 and DZNep increased IFNy-induced PDL1 expression. ChIP-qPCR analysis showed the downregulation of EZH2 decreased the $\mathrm{H} 3 \mathrm{~K} 27 \mathrm{me} 3$ levels at the CD274 promoter. These findings suggested that EZH2 regulates PD-L1 expression in hepatoma cells partly by controlling the H3K27me3 levels on the CD274 promoter. However, we observed that GSK126, a direct HMT inhibitor, and DZNep, an indirect HMT inhibitor, displayed certain differences in terms of promoting PD-L1 expression. These results suggested that 
other mechanisms might be involved in DZNep's activity, such as proteasomal degradation of PRC2 subunits, inhibition of other methylation reactions, or reactivation of thioredoxin-binding protein 2 (TXNIP), which causes disruption of PRC2 [51].

We also found that EZH2 could control the H3K27me3 level of the IRF1 promoter, which is prerequisite for IFN $\gamma$ induced upregulation of PD-L1 [52]. Downregulated expression of EZH2 significantly inhibited IFN $\gamma$-induced upregulation of IRF1. Whereas, the rescue assays showed that downregulated expression of IRF1 significantly decreased the upregulated levels of $C D 274$ promoter luciferase activity and protein expression caused by siEZH2 under IFNY stimulation. In addition, in line with the results of Lee's report [52], we identified two IRF1 response elements (IREs) on the CD274 promoter. We further identified that IRE1 was a more effective than IRE2 in IRF1mediated transcription of $C D 274$, which might reflect the higher number of IRF1 binding sites in IRE1, as shown by sequence analysis.

In addition, we also tested other inflammatory factors, such as IL- 6 and TNF $\alpha$, for their induction of PD-L1 expression on hepatoma cells. However, neither IL-6 nor TNF $\alpha$ could induce PD-L1 expression on hepatoma cells, with or without $E Z H 2$-silencing (Additional file 1: Figure S7a-d). Notably, we found TNF $\alpha$ synergistically promoted the expression of PD-L1 induced by IFNY (Additional file 1: Figure S7e). This might reflect its role of promoting the stability of PD-L1 [53]. We observed that compared with control cells, the degradation rates of ectopically expressed PD-L1 did not increase in EZH2-silenced hepatoma cells after treatment with the protein synthesis inhibitor cycloheximide $(\mathrm{CHX})$, which suggested that the regulation by EZH2 of IFN $\gamma$-induced PD-L1 does not involve the protein stability of PD-L1 in hepatoma cells (Additional file 1: Figure S8).

Drugs targeting EZH2 has been shown to promote the secretion of Th1-type chemokine and subsequent local infiltration of $\mathrm{CD}^{+} \mathrm{T}$ cells in ovarian and colon cancer [22, 23]. A recent study demonstrated a promotion role of CCRK/EZH2-NF-kB/IL-6 axis in HCC by reshaping the balance of MDSCs and $\mathrm{CD}^{+} \mathrm{T}$ cells [24]. Simultaneously blockade of CCRK and PD-L1 could inhibit MDSCs accumulation and engendered $\mathrm{CD}^{+} \mathrm{T}$ cell responses in tumor tissues, resulting in eradication of HCC. In this study, we observed that downregulating the EZH2 could enhance IFNYinduced PD-L1 expression in hepatoma cells. Survival analysis showed that patients with PD-L1 expression on TCs had poor survival in the EZH2 lowexpression group. These findings collectively suggest an important role of EZH2 in reformulating the tumor immune microenvironment.
In conclusion, the present study demonstrated that the epigenetic modifier EZH2 can suppress the expression of immune checkpoint inhibitor PD-L1 by directly upregulating the promoter $\mathrm{H} 3 \mathrm{~K} 27 \mathrm{me} 3$ levels of $C D 274$ and IRF1 in hepatoma cells and might serve as a potential therapeutic target for combination of cancer immunotherapy for immune-activated HCC.

\section{Supplementary information}

Supplementary information accompanies this paper at https://doi.org/10. 1186/s40425-019-0784-9.

Additional file 1: Figure S1. The expression of EZH2 in HCC tissue
microarray. Figure S2. Knockdown of EZH2 upregulates PD-L1 expression
induced by IFNy at different time points. Figure S3. EZH2 inhibitors pro-
motes the IFNy-induced PD-L1 expression.. Figure S4. The IFNY-STAT1
signaling is not regulated by EZH2. Figure S5. The EZH2/H3K27me3 axis
regulates the expression of IRF1. Figure S6. Transcriptome profiling and
gene ontology biological analysis of immune-suppressed and immune-
activated HCC tissues. Figure S7. Effect of IL-6 and TNFa on the expression
of PD-L1 in hepatoma cells. Figure S8. EZH2 did not effect the protein sta-
bility of PD-L1.
Additional file 2: Table S1. Patient characteristics. Table S2. siRNA
target sequences of EZH2 and IRF1 gene. Table S3. Sequences of
primers for quantitative real-time PCR. Table S4. Antibodies used in
current study. Table S5. Sequences of primers for PD-L1 promoter lucifer-
ase reporter plasmids construction. Table S6. ChIP primers used in
current study.
Additional file 3: Table S7. The genes predicted in Fig. 3c.
Additional file 4: Table S8. List of differentially expressed gene
between immune-activated and immune-suppressed HCC tissues
screened by transcriptome.

\section{Abbreviations}

ChIP: Chromatin immunoprecipitation; CHX: Cycloheximide; CXCL10: C-X-C motif chemokine ligand 10; EZH2: Enhancer of zeste 2; FACS: Fluorescence activated cell sorting; H3K27me3: Trimethylation on histone 3 lysine 27; HCC: Hepatocellular carcinoma; HMT: Histone methyltransferase; IFNy: Interferon gamma; IL-6: Interleukin 6; IRE: IRF1 response element; IRF1: Interferon regulatory factor 1; MDSCs: Myeloid-derived suppressor cells; Mo/Mys: Monocytes/macrophages; OS: Overall survival; PBS: Phosphate buffer saline; PD-1: Programmed death-1; PD-L1: Programmed death-1 ligand 1; PVDF: Polyvinylidene fluoride; qPCR: Quantitative real-time polymerase chain reaction; RFS: Relapse-free survival; RNAi: RNA interference; SDSPAGE: Sodium dodecyl sulfate-polyacrylamide gel electrophoresis; siRNA: Small interfering RNA; TCGA: The Cancer Genome Atlas; TCs: Tumor cells; TF: Transcription factor; TNFa: Tumor necrosis factor

\section{Acknowledgments}

Not applicable.

\section{Authors' contributions}

$L Z$, JX and GX conceived and designed the experiments. GX, L-LJ, C-QL, YCW and Y-MM performed the experiments. GX, L-LJ and JX analyzed the data and prepared all the figures and wrote the manuscript. Z-GZ, JC, Y-JZ and X$J Y$ provided technical support. All authors read and approved the final manuscript.

\section{Funding}

This work was supported by the National 135 Major Project of China (2017YFA0505803 and 2018ZX10302205); the National Natural Science Foundation of China (81702818, 81772536 and 81572387); the China Postdoctoral Science Foundation (2016 M592586); and the Fundamental Research Funds for the Central Universities under Grant 171gjc32. 


\section{Availability of data and materials}

The datasets used for the current study are available from the corresponding author on reasonable request.

\section{Ethics approval and consent to participate}

All authors approved and directly participated in the planning, execution and/or analysis of the data presented herein. The research presented here has been performed in accordance with the Declaration of Helsinki and has been approved by the Review Board of Sun Yat-sen University Cancer Center. The patients were informed about the sample collection and had signed informed consent forms.

\section{Consent for publication}

The content of this manuscript has not been previously published and is not under consideration for publication elsewhere.

\section{Competing interests}

The authors declare that they have no competing interests.

\begin{abstract}
Author details
${ }^{1}$ Collaborative Innovation Center of Cancer Medicine, State Key Laboratory of Oncology in South China, Sun Yat-sen University Cancer Center, Sun Yat-sen University, Guangzhou 510060, People's Republic of China. 'Department of Biliary-Pancreatic Surgery, Sun Yat-sen Memorial Hospital, Sun Yat-sen University, Guangzhou 510120, People's Republic of China. ${ }^{3}$ Guangdong Provincial Key Laboratory of Malignant Tumor Epigenetics and Gene Regulation, Sun Yat-Sen Memorial Hospital, Sun Yat-Sen University, Guangzhou 510120, People's Republic of China. ${ }^{4}$ MOE Key Laboratory of Gene Function and Regulation, State Key Laboratory of Biocontrol, School of Life Sciences, Sun Yat-sen University, Guangzhou 510275, People's Republic of China. ${ }^{5}$ Department of Hepatobiliary Oncology, Sun Yat-sen University Cancer Center, Sun Yat-sen University, Guangzhou 510060, People's Republic of China.
\end{abstract}

\section{Received: 10 April 2019 Accepted: 22 October 2019}

\section{Published online: 14 November 2019}

\section{References}

1. Naoum GE, Morkos M, Kim B, Arafat W. Novel targeted therapies and immunotherapy for advanced thyroid cancers. Mol Cancer. 2018;17:51

2. Mahoney KM, Rennert PD, Freeman GJ. Combination cancer immunotherapy and new immunomodulatory targets. Nat Rev Drug Discov. 2015;14:561-84.

3. Inarrairaegui M, Melero I, Sangro B. Immunotherapy of hepatocellular carcinoma: facts and hopes. Clin Cancer Res. 2018;24:1518-24.

4. Keenan TE, Burke KP, Van Allen EM. Genomic correlates of response to immune checkpoint blockade. Nat Med. 2019;25:389-402.

5. Topalian SL, Hodi FS, Brahmer JR, Gettinger SN, Smith DC, McDermott DF Powderly JD, Carvajal RD, Sosman JA, Atkins MB, et al. Safety, activity, and immune correlates of anti-PD-1 antibody in cancer. N Engl J Med. 2012;366: 2443-54.

6. Ribas A, Wolchok JD. Cancer immunotherapy using checkpoint blockade. Science. 2018;359:1350-5.

7. Burris HA, Infante JR, Ansell SM, Nemunaitis JJ, Weiss GR, Villalobos VM, Sikic Bl, Taylor MH, Northfelt DW, Carson WE 3rd, et al. Safety and activity of Varlilumab, a novel and first-in-class agonist anti-CD27 antibody, in patients with advanced solid tumors. J Clin Oncol. 2017:35:2028-36.

8. Lesokhin AM, Ansell SM, Armand P, Scott EC, Halwani A, Gutierrez M, Millenson MM, Cohen AD, Schuster SJ, Lebovic D, et al. Nivolumab in patients with relapsed or refractory hematologic malignancy: preliminary results of a phase lb study. J Clin Oncol. 2016;34:2698-704.

9. Duenas-Gonzalez A, Lizano M, Candelaria M, Cetina L, Arce C, Cervera E. Epigenetics of cervical cancer. An overview and therapeutic perspectives. Mol Cancer. 2005:4:38

10. Weintraub K. Take two: combining immunotherapy with epigenetic drugs to tackle cancer. Nat Med. 2016;22:8-10.

11. Emran AA, Chatterjee A, Rodger EJ, Tiffen JC, Gallagher SJ, Eccles MR, Hersey P. Targeting DNA methylation and EZH2 activity to overcome melanoma resistance to immunotherapy. Trends Immunol. 2019;40:328-44.

12. Comet I, Riising EM, Leblanc B, Helin K. Maintaining cell identity: PRC2-mediated regulation of transcription and cancer. Nat Rev Cancer. 2016;16:803-10.
13. Crea F, Hurt EM, Mathews LA, Cabarcas SM, Sun L, Marquez VE, Danesi R, Farrar WL. Pharmacologic disruption of Polycomb repressive complex 2 inhibits tumorigenicity and tumor progression in prostate cancer. Mol Cancer. 2011;10:40.

14. Hou S, Clement RL, Diallo A, Blazar BR, Rudensky AY, Sharpe AH, Sage PT. FoxP3 and Ezh2 regulate Tfr cell suppressive function and transcriptional program. J Exp Med. 2019;216:605-20.

15. Mohammad F, Weissmann S, Leblanc B, Pandey DP, Hojfeldt JW, Comet I, Zheng C, Johansen JV, Rapin N, Porse BT, et al. EZH2 is a potential therapeutic target for H3K27M-mutant pediatric gliomas. Nat Med. 2017;23:483-92

16. Jin X, Kim LY, Wu Q, Wallace LC, Prager BC, Sanvoranart T, Gimple RC, Wang $X$, Mack SC, Miller TE, et al. Targeting glioma stem cells through combined BMI1 and EZH2 inhibition. Nat Med. 2017:23:1352-61.

17. Ho TH, Kapur P, Eckel-Passow JE, Christie A, Joseph RW, Serie DJ, Cheville JC, Thompson RH, Homayoun F, Panwar V, et al. Multicenter validation of enhancer of Zeste homolog 2 expression as an independent prognostic marker in localized clear cell renal cell carcinoma. J Clin Oncol. 2017;35:3706-13.

18. Tiwari N, Tiwari VK, Waldmeier L, Balwierz PJ, Arnold P, Pachkov M, MeyerSchaller N, Schubeler D, van Nimwegen E, Christofori G. Sox4 is a master regulator of epithelial-mesenchymal transition by controlling Ezh2 expression and epigenetic reprogramming. Cancer Cell. 2013;23:768-83.

19. Li CH, To KF, Tong JH, Xiao Z, Xia T, Lai PB, Chow SC, Zhu YX, Chan SL, Marquez VE, Chen Y. Enhancer of zeste homolog 2 silences microRNA-218 in human pancreatic ductal adenocarcinoma cells by inducing formation of heterochromatin. Gastroenterology. 2013;144:1086-1097.e1089.

20. Hirukawa A, Smith HW, Zuo D, Dufour CR, Savage P, Bertos N, Johnson RM, Bui T, Bourque G, Basik M, et al. Targeting EZH2 reactivates a breast cancer subtype-specific anti-metastatic transcriptional program. Nat Commun. 2018;9:2547.

21. Ennishi D, Takata K, Beguelin W, Duns G, Mottok A, Farinha P, Bashashati A, Saberi S, Boyle M, Meissner B, et al. Molecular and genetic characterization of $\mathrm{MHC}$ deficiency identifies $\mathrm{EZH} 2$ as therapeutic target for enhancing immune recognition. Cancer Discov. 2019;9:546-63.

22. Peng D, Kryczek I, Nagarsheth N, Zhao L, Wei S, Wang W, Sun Y, Zhao E, Vatan L, Szeliga W, et al. Epigenetic silencing of TH1-type chemokines shapes tumour immunity and immunotherapy. Nature. 2015;527:249-53.

23. Nagarsheth N, Peng D, Kryczek I, Wu K, Li W, Zhao E, Zhao L, Wei S, Frankel $T$, Vatan $L$, et al. PRC2 epigenetically silences Th1-type chemokines to suppress effector T-cell trafficking in colon cancer. Cancer Res. 2016;76:275-82.

24. Zhou J, Liu M, Sun H, Feng Y, Xu L, Chan AWH, Tong JH, Wong J, Chong CCN, Lai PBS, et al. Hepatoma-intrinsic CCRK inhibition diminishes myeloidderived suppressor cell immunosuppression and enhances immunecheckpoint blockade efficacy. Gut. 2018;67:931-44.

25. Huang S, Wang Z, Zhou J, Huang J, Zhou L, Luo J, Wan YY, Long H, Zhu B. EZH2 inhibitor GSK126 suppresses antitumor immunity by driving production of myeloid-derived suppressor cells. Cancer Res. 2019;79:2009-20.

26. Herbst RS, Soria JC, Kowanetz M, Fine GD, Hamid O, Gordon MS, Sosman JA McDermott DF, Powderly JD, Gettinger SN, et al. Predictive correlates of response to the anti-PD-L1 antibody MPDL3280A in cancer patients. Nature. 2014:515:563-7.

27. Wei Y, Zhao Q, Gao Z, Lao XM, Lin WM, Chen DP, Mu M, Huang CX, Liu ZY, $\mathrm{Li} B$, et al. The local immune landscape determines tumor PD-L1 heterogeneity and sensitivity to therapy. J Clin Invest. 2019;129:3347-60.

28. Chen DP, Ning WR, Jiang ZZ, Peng ZP, Zhu LY, Zhuang SM, Kuang DM, Zheng $L$, Wu Y. Glycolytic activation of peritumoral monocytes fosters immune privilege via the PFKFB3-PD-L1 axis in human hepatocellular carcinoma. J Hepatol. 2019:71:333-43.

29. Sabbatino F, Villani V, Yearley JH, Deshpande V, Cai L, Konstantinidis IT, Moon C, Nota S, Wang Y, Al-Sukaini A, et al. PD-L1 and HLA class I antigen expression and clinical course of the disease in intrahepatic Cholangiocarcinoma. Clin Cancer Res. 2016;22:470-8.

30. Sun C, Mezzadra R, Schumacher TN. Regulation and function of the PD-L1 checkpoint. Immunity. 2018;48:434-52.

31. Jiang X, Wang J, Deng X, Xiong F, Ge J, Xiang B, Wu X, Ma J, Zhou M, Li X, et al. Role of the tumor microenvironment in PD-L1/PD-1-mediated tumor immune escape. Mol Cancer. 2019;18:10.

32. Liu CQ, Xu J, Zhou ZG, Jin LL, Yu XJ, Xiao G, Lin J, Zhuang SM, Zhang YJ, Zheng $L$. Expression patterns of programmed death ligand 1 correlate with different microenvironments and patient prognosis in hepatocellular carcinoma. Br J Cancer. 2018;119:80-8. 
33. Kuang DM, Peng C, Zhao Q, Wu Y, Chen MS, Zheng L. Activated monocytes in peritumoral stroma of hepatocellular carcinoma promote expansion of memory T helper 17 cells. Hepatology. 2010;51:154-64.

34. Moon JW, Kong SK, Kim BS, Kim HJ, Lim H, Noh K, Kim Y, Choi JW, Lee JH, Kim YS. IFNgamma induces PD-L1 overexpression by JAK2/STAT1/RF-1 signaling in EBV-positive gastric carcinoma. Sci Rep. 2017;7:17810.

35. Mandai M, Hamanishi J, Abiko K, Matsumura N, Baba T, Konishi I. Dual faces of IFNgamma in cancer progression: a role of PD-L1 induction in the determination of pro- and antitumor immunity. Clin Cancer Res. 2016;22: 2329-34.

36. Kim J, Shin Y, Lee S, Kim M, Punj V, Lu JF, Shin H, Kim K, Ulmer TS, Koh J, et al. Regulation of breast cancer-induced osteoclastogenesis by MacroH2A1.2 involving EZH2-mediated H3K27me3. Cell Rep. 2018;24:224-37.

37. Tan J, Yang $X$, Zhuang L, Jiang $X$, Chen W, Lee PL, Karuturi RK, Tan PB, Liu ET, Yu Q. Pharmacologic disruption of Polycomb-repressive complex 2mediated gene repression selectively induces apoptosis in cancer cells. Genes Dev. 2007;21:1050-63.

38. McCabe MT, Ott HM, Ganji G, Korenchuk S, Thompson C, Van Aller GS, Liu Y, Graves AP, Della Pietra A 3rd, Diaz E, et al. EZH2 inhibition as a therapeutic strategy for lymphoma with EZH2-activating mutations. Nature. 2012;492: $108-12$

39. Vire E, Brenner C, Deplus R, Blanchon L, Fraga M, Didelot C, Morey L, Van Eynde A, Bernard D, Vanderwinden JM, et al. The Polycomb group protein EZH2 directly controls DNA methylation. Nature. 2006;439:871-4.

40. Li LC, Dahiya R. MethPrimer: designing primers for methylation PCRs. Bioinformatics. 2002;18:1427-31.

41. Farre $D$, Roset R, Huerta M, Adsuara JE, Rosello L, Alba MM, Messeguer X Identification of patterns in biological sequences at the ALGGEN server: PROMO and MALGEN. Nucleic Acids Res. 2003;31:3651-3.

42. Messeguer X, Escudero R, Farre D, Nunez O, Martinez J, Alba MM. PROMO: detection of known transcription regulatory elements using species-tailored searches. Bioinformatics. 2002;18:333-4.

43. Cerami E, Gao J, Dogrusoz U, Gross BE, Sumer SO, Aksoy BA, Jacobsen A, Byrne CJ, Heuer ML, Larsson E, et al. The cBio cancer genomics portal: an open platform for exploring multidimensional cancer genomics data. Cancer Discov. 2012;2:401-4.

44. Gao J, Aksoy BA, Dogrusoz U, Dresdner G, Gross B, Sumer SO, Sun Y, Jacobsen A, Sinha R, Larsson $E$, et al. Integrative analysis of complex cancer genomics and clinical profiles using the cBioPortal. Sci Signal. 2013;6:pl1.

45. Loo Yau H, Ettayebi I, De Carvalho DD. The cancer epigenome: exploiting its vulnerabilities for immunotherapy. Trends Cell Biol. 2019;29:31-43.

46. Zhao Y, Ding L, Wang D, Ye Z, He Y, Ma L, Zhu R, Pan Y, Wu Q, Pang K, et al. EZH2 cooperates with gain-of-function p53 mutants to promote cancer growth and metastasis. EMBO J. 2019;38:e99599.

47. Cai MY, Tong ZT, Zheng F, Liao YJ, Wang Y, Rao HL, Chen YC, Wu QL, Liu YH, Guan XY, et al. EZH2 protein: a promising immunomarker for the detection of hepatocellular carcinomas in liver needle biopsies. Gut. 2011; 60:967-76

48. Wee ZN, Li Z, Lee PL, Lee ST, Lim YP, Yu Q. EZH2-mediated inactivation of IFN-gamma-JAK-STAT1 signaling is an effective therapeutic target in MYCdriven prostate cancer. Cell Rep. 2014;8:204-16.

49. Toyokawa G, Takada K, Tagawa T, Hamamoto R, Yamada Y, Shimokawa M, Oda Y, Maehara Y. A positive correlation between the EZH2 and PD-L1 expression in resected lung adenocarcinomas. Ann Thorac Surg. 2019;107: 393-400.

50. Asgarova A, Asgarov K, Godet Y, Peixoto P, Nadaradjane A, Boyer-Guittaut M, Galaine J, Guenat D, Mougey V, Perrard J, et al. PD-L1 expression is regulated by both DNA methylation and NF-KB during EMT signaling in non-small cell lung carcinoma. Oncoimmunology. 2018;7:e1423170.

51. Zhou J, Bi C, Cheong LL, Mahara S, Liu SC, Tay KG, Koh TL, Yu Q, Chng WJ. The histone methyltransferase inhibitor, DZNep, up-regulates TXNIP, increases ROS production, and targets leukemia cells in AML. Blood. 2011; 118:2830-9.

52. Lee SJ, Jang BC, Lee SW, Yang YI, Suh SI, Park YM, Oh S, Shin JG, Yao S, Chen $L$, Choi $\mathbb{I H}$. Interferon regulatory factor-1 is prerequisite to the constitutive expression and IFN-gamma-induced upregulation of B7-H1 (CD274). FEBS Lett. 2006;580:755-62.

53. Lim SO, Li CW, Xia W, Cha JH, Chan LC, Wu Y, Chang SS, Lin WC, Hsu JM, Hsu YH, et al. Deubiquitination and stabilization of PD-L1 by CSN5. Cancer Cell. 2016:30:925-39.

\section{Publisher's Note}

Springer Nature remains neutral with regard to jurisdictional claims in published maps and institutional affiliations.

\section{Ready to submit your research? Choose BMC and benefit from:}

- fast, convenient online submission

- thorough peer review by experienced researchers in your field

- rapid publication on acceptance

- support for research data, including large and complex data types

- gold Open Access which fosters wider collaboration and increased citations

- maximum visibility for your research: over $100 \mathrm{M}$ website views per year

At BMC, research is always in progress.

Learn more biomedcentral.com/submissions 